\title{
Academic expertise in assisting private companies in the fields of environment and environmental toxicology: the role of individual expertise
}

\author{
Christian Mougin ${ }^{1,2}$ (D) Peter G. C. Campbell ${ }^{3} \cdot$ Michel Couderchet $^{4} \cdot$ Patrice Denèfle $^{5} \cdot$ Fabrice Martin-Laurent $^{6}$. \\ Philippe Roland ${ }^{7} \cdot$ Vera I. Slaveykova ${ }^{8} \cdot$ Tatiana Vincent $^{9} \cdot$ Delphine Delaunay $^{10}$
}

Received: 13 July 2020 / Accepted: 10 November 2020 / Published online: 14 November 2020

(C) Springer-Verlag GmbH Germany, part of Springer Nature 2020

\begin{abstract}
The scientific knowledge produced by academic research can be valued in all sectors of human activity, including private sector. The ROVALTAIN Foundation organized a round-table during its scientific day in 2019. It crossed the points of view of academic scientists and industrial partners, addressing five main topics. The first one concerned the validation of a common definition of the academic research/private partners interface. Then, the group discussed the place for academic expertise in the corporate world; the advantages of involving academic researchers in expertise for the private sector; and the limits of this model. To conclude, the need of a third party, like the ROVALTAIN Foundation, as a catalyzer in building the interface between academic research and private partners has been discussed.
\end{abstract}

Keywords Ecotoxicology $\cdot$ Toxicology $\cdot$ Health $\cdot$ Expertise $\cdot$ Academic research $\cdot$ Private companies

\section{Introduction}

Scientific expertise is defined as "the expression of knowledge formulated in response to a request from those who have a decision to make, knowing that this response is intended to be integrated into a decision process" (INRA 2011; Roqueplo 1997). In this context, the scientific knowledge produced by academic research can be valued in all sectors of human

Responsible Editor: Philippe Garrigues

Christian Mougin

christian.mougin@inrae.fr

1 Université Paris-Saclay, INRAE, AgroParisTech, UMR ECOSYS, Plateforme Biochem-Env, F-78026 Versailles, France

2 CNRS, Sorbonne Université, Federation Ile-de-France of Research for the Environment FR3020, F-75005 Paris, France

3 Centre Eau Terre et Environnement, Institut national de la Recherche scientifique, Université du Québec, 490 de la Couronne, Québec, QC G1K 9A9, Canada

4 Unité de Recherche Vignes et Vin de Champagne, Université de Reims Champagne-Ardenne, EA 4707, F-51687 Reims Cedex 2, France activity, thanks to the numerous interfaces of academics with the different components of the society, including the private sector.

The ROVALTAIN Foundation is a nonprofit organization dedicated to scientific cooperation in the Environmental field. The main mission of the foundation is to help moving towards a pollution-free world, which it chooses to achieve by supporting research in Health and Environment with a strong

Université Paris Descartes et ParisTech, F-75006 Paris, France

6 INRAE, AgroSup Dijon, Université de Bourgogne, UMR Agroécologie, F-21065 Dijon, France

7 Renault Automobiles, F-78280 Guyancourt, France

8 Department F.-A. Forel for Environmental and Aquatic Sciences, Environmental Biogeochemistry and Ecotoxicology, Faculty of Sciences, Earth and Environment Sciences, Uni Carl Vogt, University of Geneva, 66 Blvd Carl-Vogt, CH-1211 Geneva, Switzerland

9 k53 production, 185 impasse du mûrier, F-26450 Charols, France

10 ROVALTAIN Foundation, 12 Cours Emilie du Châtelet, F-26300 Alixan, France 
orientation towards the fields of ecotoxicology and environmental toxicology and by ensuring the up-to-date dissemination of these research results, towards the citizens. The foundation also has the mission to favour synergies between the various actors in the health-environment field (researchers, elected representatives, companies, citizens, farmers, governmental and non-governmental institutions) to facilitate the dialogue and the setting up of concrete and efficient partnerships. One main action of the foundation is to assist private companies by providing them innovative training courses and top-notch scientific partners when the companies are in need of scientific expertise.

In this paper, we report the points of discussion addressed during the course of the round-table "academic expertise in assisting private companies" which was held during the ROVALTAIN Foundation on November 15, 2019, in Alixan, France. This round-table focused on scientific expertise intended for the business world and brought together experts from both academia and industry to consider the advantages and limitations encountered in this particular type of collaborative framework.

\section{What is the academic research/private partners interface: a relationship of trust, a common vision?}

Academic expertise resulting from researches funded by public funding, industry or raised funds from the public is essential for knowledge and technology transfer (Kotiranta et al. 2020). Depending on the objectives involved, they take many forms (Boardman and Ponomaroiv 2009). Institutionalized or collective, it can most often shed scientific light on a given subject based on critical analysis and the synthesis of international peer-reviewed literature. Individuals can carry out the expertise by connecting an academic scientist with a private partner to meet an identified need. On the industrial side, the practical vision of both fundamental applied research work as well as the mastering of technical and commercial stages guarantee value creation.

During the round-table, the important place that individual expertise of researchers should take in relations between academic research and private partners in the coming years was acknowledged. Carrying out any project between partners of different origins and skills requires overcoming their isolation in order to find collaborative solutions. The use of individual academic expertise makes it possible to bring together a field of in-depth knowledge and industrial or societal know-how. It allows a better understanding of the constraints and expectations of each of the parties. "You have to formulate the right question to find the right solution". Often, companies encapsulated in hermetic silos identify the problem correctly but struggle to ask the right questions to solve it. Likewise, academic researchers are focused on the scientific valorization of their discoveries, but most of them have not always in mind the application potential of their work. Helping and supporting companies to formulate the right questions and develop creative answers amounts to co-building a common vision that is the first keystone of new partnership model between academic and private research. This co-construction is not limited to the concepts of the one who knows/the creator opposed to the learner/payer, or even to a customer/supplier relationship, but rather results from a real cooperative work that leads to a unique and original solution enriching both parties. In addition, exchanges of processes and management from industry to academics and vice versa is a real added value to publicly funded research that benefits to society.

An advance in the "path of innovation" then relies on a real interface established by shared expertise and knowledge. However, a private company, more than an academic partner, is looking for a profit in the more or less long term and may retain part of the information to avoid loss of expertise and leadership, until it is certain to receive this benefit. To resolve this problem, the signature of a non-disclosure agreement is highly recommended at the start of the appraisal, followed by a research and development contract that explicitly defines the input of each partner and the outlines of the collaboration and stipulates the distribution of intellectual property. Despite these contractual aspects, which are necessary to define the partnership relations, it is important to increase mutual trust between the two parties to develop an effective win-win strategy.

\section{What place for academic expertise in the corporate world?}

Academic expertise finds its place when answering a precise, punctual question for the company, which does not necessarily have the resources to address it internally. It also makes it possible to explore more forward-looking avenues, for example, upstream of regulations, in order to anticipate changes before they become necessary. Gaps can exist between current or future regulations and knowledge, for example, on the use of chemical compounds (active substances). In that case, it is important that the company, whose know-how is at the heart of its strategy and therefore uses a strategic watch on all technological and regulatory fields, can be assisted in the implementation of regulations by providing scientific data to support its reasoning. Many manufacturers are directly concerned today by the impact of their activities on the environment and on health and wish to be supported in the implementation of long-term action plans. Individual expertise can turn into long-term collaboration between manufacturers and researchers from different laboratories. 
Academic expertise also finds its place in the corporate world by bringing in rare skills. This is often the case in the medical sector, where the need for independence reduces the choice of competent external experts. Even if one has to keep in mind the question posed by the "revolving door", excessive prudence in the exercise of the precautionary principle aimed at avoiding any potential conflict of interest can thus lead regulatory agencies to seek opinions from inexperienced and under-qualified evaluators, which leads to absurd situations, especially during the management of health crises. Here again, we must trust expert researchers in their capacity to give informed opinions while regulating the actions of lobbyists with more transparency.

In addition, a survey of US university scientists revealed a synergy between a wide range of academic activities and roles and interactions with the private sector (Boardman and Ponomaroiv 2009).

\section{What are the advantages of involving academic researchers in expertise for the private sector?}

The development of scientific expertise for the private sector boosts the career of scientists by offering new opportunities and developing networks. They are required to build cuttingedge projects in response to calls open for funding. However, the value of an appraisal activity linked to the business world is perceived differently depending on the seniority (or age) of the expert, and the country. When they become experts, scientists find themselves working according to different rules and in different contexts than when they carry out their activity as academic researchers (Maxim and Arnold 2012). Today, in research institutes, carrying out expertise for regulatory bodies (government agencies, French Agency for Food, Environmental and Occupational Health \& Safety (ANSES), European Food Safety Authority (EFSA)...; standardization structures, French Association for Standardization (AFNOR), International Organization for Standardization (ISO)...) is recognized by the evaluation board. Being part of a working group or expertise at national or international levels appears prestigious and can contribute to the promotion of the career of scientists. This recognition is much less true when we speak of academic scientific expertise towards private sector companies, which is still viewed as the dark side by a part of the scientific community. This perception shared by society is reinforced by various scandals involving scientists with questionable ethical practices. In Quebec, for example, the experience of an academic expert is considered to be a potential resource for the community, and it is thus taken into account in the professorial evaluations. This is an integral part of the academic curriculum. Unlike many European countries, North American countries have fully integrated the value and importance of the contribution of the scientific expertise of academic researchers to industrial development.

Furthermore, the benefits received are reciprocal. It should not be forgotten that this type of expertise allows the academic researchers to confront the concerns of the socio-economic world and to get in touch with other management and process strategies, by getting out of their "ivory tower". This sometimes destabilizing yet enriching experience, which also favours the dissemination of the finalized benefits of academic works, is then an asset, which can, and must, be transferred to students in training. In a second step, the relationship forged with industry personnel can be sustained over time and lead to the achievement of big scientific projects, which can be the opportunity to set up shared industry-oriented PhDs partly funded by public grants (CIFRE programme in France). These collaborations transcend the boundaries between the academic and private sectors and facilitate transitions from one sector to another for young graduates. Like private research, academic research is bound to innovate. Financing innovation, in particular the acquisition of new equipment, can also go through a partnership with the private sector.

Despite everything, if certain scientific tools and platforms, for example, grouped in research infrastructures (national and international, i.e.; Biochem-Env, http://www.biochemenv.fr), are by nature open to partnerships with the private sector, we must however be careful not to limit academic research to collaborative research programmes with the private sector. An equilibrium has to be found in which academic research has to remain able to work in a free, autonomous manner and have its own research avenues. This depends upon researchers but mainly on political choice to support public research to lead to improve scientific knowledge, which is the source of innovations promoting the competitiveness of our society. Research activities financed without compensation (i.e. patronage) can be foreseen when public money is lacking, but scientific partnerships or a co-creation activity must systematically be preferred, in particular to service contracts. The most reliable indicators of co-creation activity are the percentage of co-ownership of patents or the co-signature of possible research output including scientific articles and conference presentations.

\section{What are the limits of the academic world involvement in expertise towards the private sector?}

For various reasons, the scientific culture is often being restricted to the "knowledge spheres" of the academic and private sectors. Knowledge still percolates poorly towards the different components of society (citizens, students, politicians, etc.), which can be an obstacle to the involvement of the academic sector outside its usual sphere. The mixture of the two 
cultures, academic and private, also finds its limits due to the suspicious positioning of society towards academic researchers, when they interact with a private society. This partnership often generates a perception of loss of independence or conflict of interest, which results in a loss of confidence and consideration, sometimes relayed by peers. Therefore, the excessive use of the precautionary principle can lead to a real problem of availability of competent so-called "independent" experts in the evaluation of a partnership project by the authorities.

This partnership conducted on an individual basis can also generate obstacles to communication and scientific publication (confidentiality clauses, embargoes on data, which are known and mentioned in the contract signed by both parties). That fact can have negative consequences, not only on the career of scientists but also (and above all) on the careers of the involved students (doctoral and post-doctoral) who need to publish quickly to expand their $\mathrm{CV}$ if they decide to embark on an academic career.

\section{What could be the role for the ROVALTAIN Foundation in building the interface between academic research and private partners?}

A third-party structure such as the ROVALTAIN Foundation in ecotoxicology/toxicology can act as a catalyst to reduce invisible but existing barriers (often cultural) between the academic research/private sector in order to develop a win-win partnership for a priori and a posteriori assessment of the risks posed by different anthropogenic activities on environmental and human health. By positioning itself as a network pilot, facilitator and one-stop shop, and through its knowledge of both the academic world and the private sector, the foundation can help define business issues, facilitate communication among players and offer the most relevant help to mobilize funding and incubators that will speed up the interaction process. It can also contribute to the administrative support.

Acknowledgements The authors thank the ROVALTAIN Foundation (Alixan, France) for organizing this round-table meeting. Biochem-Env (https://doi.org/10.15454/HA6V6Y) is a service of the investment d'Avenir infrastructure AnaEE-France, overseen by the French National Research Agency (ANR-11-INBS-0001). Delphine Delaunay, Fabrice Martin-Laurent and Christian Mougin are members of the ECOTOX network (https://www6.inrae.fr/ecotox/).

\section{Compliance with ethical standards}

Disclaimer The views and opinions expressed in this article are those of the authors and do not necessarily reflect the official policy of organization, employer or company.

\section{References}

Boardman PC, Ponomaroiv BL (2009) University researchers working with private companies. Technovation 29:142-153

INRA (2011) Charte INRA de l'Expertise Scientifique Institutionnelle.https:/www.inrae.fr/sites/default/files/pdf/ 885e2467ea09c7f4ee05f928003d3c51.pdf. Accessed 14 Nov 2020

Kotiranta A, Tahvanainen A, Kovalainen A, Poutanen S (2020) Forms and varieties of research and industry collaboration across disciplines. Heliyon 6(3):e03404

Maxim L, Arnold G (2012) Entre recherche académique et expertise scientifique : des mondes de chercheurs. Hermès, La Revue 2012/ 3 (64), 9-13

Roqueplo P (1997) Entre savoir et décision, l'expertise scientifique. Collection Sciences en Question, Editions Quae, 112 pages, EAN13 9782738007131

Publisher's note Springer Nature remains neutral with regard to jurisdictional claims in published maps and institutional affiliations. 